\title{
Decreased MiR-155 Level in the Peripheral Blood of Non-Alcoholic Fatty Liver Disease Patients may Serve as a Biomarker and may Influence LXR Activity
}

\author{
Lei Wang $^{\mathrm{a}}$ Ning Zhang ${ }^{\mathrm{b}}$ Zun Wanga ${ }^{\mathrm{a}}$ Dong-mei Aia Zhen-yu Cao $^{\mathrm{a}}$ \\ Hua-ping Panc
}

aDepartment of Rehabilitation Medicine, Second Medical School of Nanjing University of Chinese Medicine, Nanjing, bepartment of Cardiology, Nanjing Drum Tower Hospital, Nanjing University Medical School, Nanjing 'Department of Rehabilitation Medicine, The Affiliated Jiangning Hospital of Nanjing Medical University, Nanjing, China

\section{Key Words}

miR-155 • LXR $\alpha$ NAFLD $\cdot$ Peripheral blood

\begin{abstract}
Background: Obesity is now a common risk factor for non-alcoholic fatty liver disease (NAFLD). Thus, it is important to explore its underlying mechanisms. Methods: Total RNA was extracted from peripheral whole blood samples from 50 NAFLD patients and 50 healthy controls. In addition, human liver specimens were obtained through liver biopsies from NAFLD patients and healthy controls. The level of miRNA was studied using real-time PCR. The expression of lipogenic genes was analyzed using western blot, and a dual luciferase reporter assay was conducted to identify the possible target gene. Adenovirus vectors were injected into the tail vein of the high fat diet (HFD)-fed mice to study the role of miR-155 on lipid accumulation in vivo. Results: The level of miR-155 was markedly reduced in the livers and peripheral blood of NAFLD patients compared with healthy controls. Upregulation of miR-155 decreased intracellular lipid content and the SREBP1 and FAS protein levels, while inhibition of miR-155 enhanced the intracellular lipid content. The dual luciferase reporter assay showed that Liver $X$ receptor (LXR) $\alpha$ was the target gene of miR-155, and silencing miR-155 reduced the expression of SREBP1 and FAS. An in vivo study showed that upregulation of miR-155 decreased the hepatic lipid accumulation mainly by suppressing the LXR $\alpha$-dependent lipogenic signaling pathway. Conclusions: In summary, decreased expression of miR-155 in the peripheral blood may be utilized as a potential novel biomarker for NAFLD screening mainly by targeting LXR $\alpha$.
\end{abstract}




\section{Cellular Physiology Cell Physiol Biochem 2016;39:2239-2248

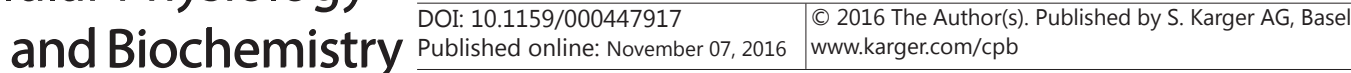 \\ Wang et al.: MiR-155 Targets LXR $\alpha$ in NAFLD Livers}

\section{Introduction}

At present, obesity is a worldwide health problem and continues to increase due to changes in dietary habits and sedentary lifestyles [1]. Moreover, obesity is a common risk factor for non-alcoholic fatty liver disease (NAFLD), which causes a chronic hepatic pathology and affects numerous people around the world [1-3]. Thus, it is important to develop effective biomarkers for screening the populations at risk.

The liver is an important organ that regulates glycogen deposition, lipogenesis, fatty acid oxidation and cholesterol transport [4]. Liver X receptor (LXR) belongs to the nuclear receptor superfamily of ligand-activated transcription factors $[4,5]$. Two major isoforms have been identified for LXR, including LXR $\alpha$ and $\operatorname{LXR} \beta[4,6]$. Through interaction with the endogenous or synthetic ligands, LXR $\alpha$ plays a key role in the liver. LXR $\alpha$ can bind the retinoid $\mathrm{X}$ receptor (RXR), thereby activating the transcription of downstream genes [7]. In the liver, LXR $\alpha$ is found to activate SREBP-1 gene transcription, which then enhances the subsequent activation of lipogenic genes, including fatty acid synthase (FAS), acetyl-CoA carboxylase (ACC1), and stearoyl-coenzyme A desaturase (SCD-1) [8].

MicroRNAs (miRNAs) are a class of short, noncoding RNAs that widely modulate gene expression $[9,10]$. Initially, microRNAs are transcribed as long primary transcripts and then processed by the ribonuclease III endonucleases Drosha and Dicer to form mature 22-nucleotide non-coding small RNAs. Through a base-pairing mechanism, the mature miRNAs can bind to the $3^{\prime}$-untranslated region (UTR) of their target mRNAs, resulting in mRNA degradation [11]. Abnormal expression of miRNAs has been widely identified in different physiological and pathological circumstances [12-14]. For instance, miR-291b-3p was recently found to prompt hepatic lipogenesis mainly by targeting AMPK $\alpha 1$, and miR-125b enhances lipogenesis through binding the 3'UTR of SCD-1 $[12,13]$. In the current study, we mainly focus on miR-155, which has been widely explored in tumors $[15,16]$. For instance, ESAT-6 secreted by Mycobacterium Tuberculosis was reported to enhance the apoptosis of macrophages via disturbing the microRNA155-SOCS1 interaction [17]. However, its specific role in NAFLD has never been explored. Here, we first demonstrated that the level of miR-155 was significantly decreased in the livers and peripheral blood of NAFLD patients. Further study revealed that reduction of miR-155 enhanced hepatic lipogenesis mainly by binding the 3'UTR of LXR $\alpha$.

\section{Materials and Methods}

\section{Human liver specimens}

All peripheral whole blood samples of NAFLD patients $(n=50)$ and healthy individuals $(n=50)$ were obtained from the Second Medical School of Nanjing University of Chinese Medicine between May 2015 and January 2016. The human liver biopsies and clinical procedures were performed with patient consent within the diagnostic workup of NAFLD.

For liver biopsies, a total of 11 patients diagnosed with NAFLD and 11 healthy control subjects were included in the study. Physical examinations, biochemical measurements, and body mass index (BMI) calculations were performed on all of the study participants.

The application for patient-derived materials was approved by the Research Ethics Committee of Second Medical School of Nanjing University of Chinese Medicine, and written consent was obtained from all of the patients.

\section{Animal treatment}

Four-week-old wild-type (WT) C57BL/6J mice were purchased from the Peking University Health Science Center. C57BL/6J mice were fed a standard chow diet or a high fat diet (HFD) for 10 weeks in a temperature- $\left(20-24^{\circ} \mathrm{C}\right)$ and humidity-controlled (45-55\%) environment. A $12 \mathrm{~h} / 12 \mathrm{~h}$ light/dark cycle was maintained for the animal housing rooms. HFD-fed mice were injected intravenously through the tail vein with an adenovirus expressing miR-155 inhibitor (Ad-155i) or a negative control (Ad-Con) adenoviral vector for 1 week. On day 7 after adenovirus injection, the mice were anesthetized, and blood was collected via cardiac 


\section{Cellular Physiology Cell Physiol Biochem 2016;39:2239-2248 \begin{tabular}{l|l|l} 
and Biochemistry Published online: November 07, 2016 & $\begin{array}{l}\text { C } 2016 \text { The Author(s). Published by S. Karger AG, Basel } \\
\text { www.karger.com/cpb }\end{array}$
\end{tabular} \\ Wang et al.: MiR-155 Targets LXR $\alpha$ in NAFLD Livers}

puncture. Livers were harvested, snap-frozen in liquid nitrogen, and stored at $-80^{\circ} \mathrm{C}$ for further analysis [18]. All of the mouse procedures were approved by the animal committee of the Second Medical School of Nanjing University of Chinese Medicine.

\section{Adenoviral vector construction}

Recombinant adenoviruses expressing miR-155 inhibitor (Ad-miR-155-i) and the negative control (NC) adenovirus vector containing GFP (Ad-Con) were custom made by Shanghai Genechem Co., Ltd.

\section{Cell culture}

Murine liver cell line Hep1-6 (American Type Culture Collection) was cultured in Dulbecco's modified Eagle medium (low glucose; Invitrogen) supplemented with $10 \%$ fetal bovine serum (FBS, Hyclone), 100 units $/ \mathrm{ml}$ penicillin (Invitrogen) and $0.1 \mathrm{mg} / \mathrm{ml}$ streptomycin (Hyclone) at $37^{\circ} \mathrm{C}$ with humidified air and $5 \%$ $\mathrm{CO}_{2}$.

RNA isolation and real-time PCR

The total RNA from the whole blood samples $(5 \mathrm{ml})$ collected in tubes containing EDTA was rigorously extracted with RNAVzol LS (Vigorous Biotechnology, Beijing) according to the manufacturer's instructions. Stem-loop reverse transcription-polymerase chain reaction (RT-PCR) was conducted with the samples to detect and quantify mature miRNAs by using stem-loop antisense primer mix and M-MuLV transcriptase (NEB).

The SYBR Green I method was used for real-time PCR with the Bio-Rad iQ5 system (Bio-Rad) according to the manufacturer's instructions (TaKaRa) The relative expression level of a miRNA was normalized to an internal invariant control, U6 small nucleolar RNA. Each reaction was performed in triplicate, and analysis was performed using the $2^{-\Delta \Delta C T}$ method.

\section{Western blot analysis}

A total of $15 \mu \mathrm{g}$ protein was separated by 10\% SDS-PAGE and then transferred to PVDF membrane (Millipore), followed by 8\% nonfat dry milk blocking. After washing with PBST three times (5 min/wash), the membranes were incubated with primary antibodies at $4^{\circ} \mathrm{C}$ overnight. The blot was incubated with HRP-conjugated anti-IgG, followed by detection with ECL (Millipore). Antibodies against LXR $\alpha$ (ab28478) were purchased from Abcam. FAS (\#3180) and $\beta$-actin (\#4970) were obtained from Cell Signaling Technology. An antibody against SREBP1 was purchased from Santa Cruz Biotechnology (sc-366).

\section{Triglyceride measurement}

The content of tissue triglycerides was measured as described previously $[18,19,20]$ using a triglyceride enzymatic assay kit (ShenSuoYouFu Medical Diagnostic Products Co., Ltd., Shanghai, China).

Histological analysis of tissues

Frozen sections of livers were paraformaldehyde-fixed for Oil red $\mathrm{O}$ or hematoxylin and eosin (H\&E) staining.

\section{Luciferase reporter assay}

The 3'UTR sequence of $L X R \alpha$ predicted to interact with miR-155 was cloned from the genomic DNA of the Hep1-6 cell line and inserted into the Xbal and Sacl sites of the pmir-GLO control luciferase reporter vector (Promega, Madison, WI). HEK293 cells were co-transfected with internal control vector pmir-GLO (Promega), pmir-GLO- SREBP1 3'UTR and miR-155 mimics (or negative control) using Effectene (Qiagen) for $48 \mathrm{~h}$. Luciferase reporter assays were performed using the Dual-Glo Luciferase assay system according to standard protocols (Promega).

\section{Statistics}

The data are represented as means \pm standard error of the mean (SEM). Differences were analyzed by Student's $t$-test with significance set at $P<0.05$. 


\section{Cellular Physiology Cell Physiol Biochem 2016;39:2239-2248 \begin{tabular}{l|l|l} 
and Biochemistry $10.1159 / 000447917$ & (c) 2016 The Author(s). Published by S. Karger AG, Basel
\end{tabular} \\ Wang et al.: MiR-155 Targets LXR $\alpha$ in NAFLD Livers}

\section{Results}

\section{Decreased level of miR-155 in the peripheral blood of NAFLD patients}

The demographics and clinical characteristics of the healthy controls and NAFLD patients are outlined in Table 1 . The age distribution was similar in the three groups $(P>0.05)$. H\&E staining showed large vacuoles, which were the lipid droplets in the livers of NAFLD patients (Fig. 1A). Real-time PCR analysis demonstrated that the expression of miR-155 was significantly decreased in the livers of NAFLD patients (Fig. 1B). More importantly, we also examined the expression of miR-155 in the peripheral blood of NAFLD patients and healthy control subjects using real-time PCR. As shown in Fig. 1C, the expression of miR-155 was significantly suppressed in the peripheral blood of NAFLD patients compared with that of healthy controls $(\mathrm{P}<0.001)$. The relative expression of miR-155 was $0.13 \pm 0.045$ for NAFLD patients and $1 \pm 0.35$ for healthy controls. Furthermore, we have also tested the expression of LXR-sensitive genes in the livers of NAFLD patients who have a reduced miR-155 level. According to our analysis,

Table 1. Clinical and biochemical characteristics of healthy controls and patients with nonalcoholic fatty liver disease $n(\%)$

\begin{tabular}{llll}
\hline Characteristic & Control $(\mathrm{n}=50)$ & NAFLD $(\mathrm{n}=50)$ & P value \\
\hline Gender (males/females) & $27 / 23$ & $25 / 25$ & - \\
Age (yr) & $47.0 \pm 9.3$ & $46.5 \pm 9.8$ & 0.93 \\
BMI $\left(\mathrm{kg} / \mathrm{m}^{2}\right.$ ) & $21.0 \pm 4.9$ & $27.4 \pm 3.1$ & 0.03 \\
Smoking & no & no & - \\
Waist circumference (cm) & $75.4 \pm 8.6$ & $96.2 \pm 9.7$ & 0.001 \\
Diabetes Mellitus & no & no & - \\
Metabolic syndrome & no & no & - \\
Hypertension & no & no & - \\
Systolic blood pressure (mmHg) & $107.2 \pm 6.5$ & $115.4 \pm 6.3$ & 0.04 \\
Diastolic blood pressure (mmHg) & $75.3 \pm 4.5$ & $77.3 \pm 6.5$ & 0.14 \\
AST median (min-max, U/L) & $17.1(13-24)$ & $18.4(12-26)$ & 0.43 \\
ALT median(min-max, U/L) & $14.8(8-23)$ & $22.7(11-38)$ & 0.02 \\
Total cholesterol (mmol/L) & $4.8 \pm 0.4$ & $5.2 \pm 1.1$ & 0.13 \\
HDL-cholesterol (mmol/L) & $1.5 \pm 0.3$ & $1.3 \pm 0.2$ & 0.09 \\
LDL-cholesterol (mmol/L) & $2.0 \pm 0.7$ & $2.9 \pm 1.3$ & 0.07 \\
Triglycerides (mmol/L) & $0.8 \pm 0.3$ & $2.3 \pm 1.7$ & 0.008 \\
\hline
\end{tabular}

Fig. 1. The level of miR-155 was significantly decreased in the peripheral blood of NAFLD patients compared with that of healthy controls. (A) H\&E staining showed large vacuoles in the livers of NAFLD patients. (B) Real-time PCR analysis demonstrated that the expression of miR-155 was significantly decreased in the livers of NAFLD patients $(n=11)$ compared with healthy controls $(n=11)$. (C) The expression of miR-155 was significantly suppressed in the peripheral blood of NAFLD patients $(n=50)$ compared with that of healthy control individuals $(n=50)$. (D) The levels of genes-related-lipogenesis were significantly upregulated in the livers of NAFLD patients $(n=11)$ compared with healthy controls $(n=11)$. Data represent the mean \pm S.D. ${ }^{* * *} \mathrm{p}<0.001$ vs. controls.

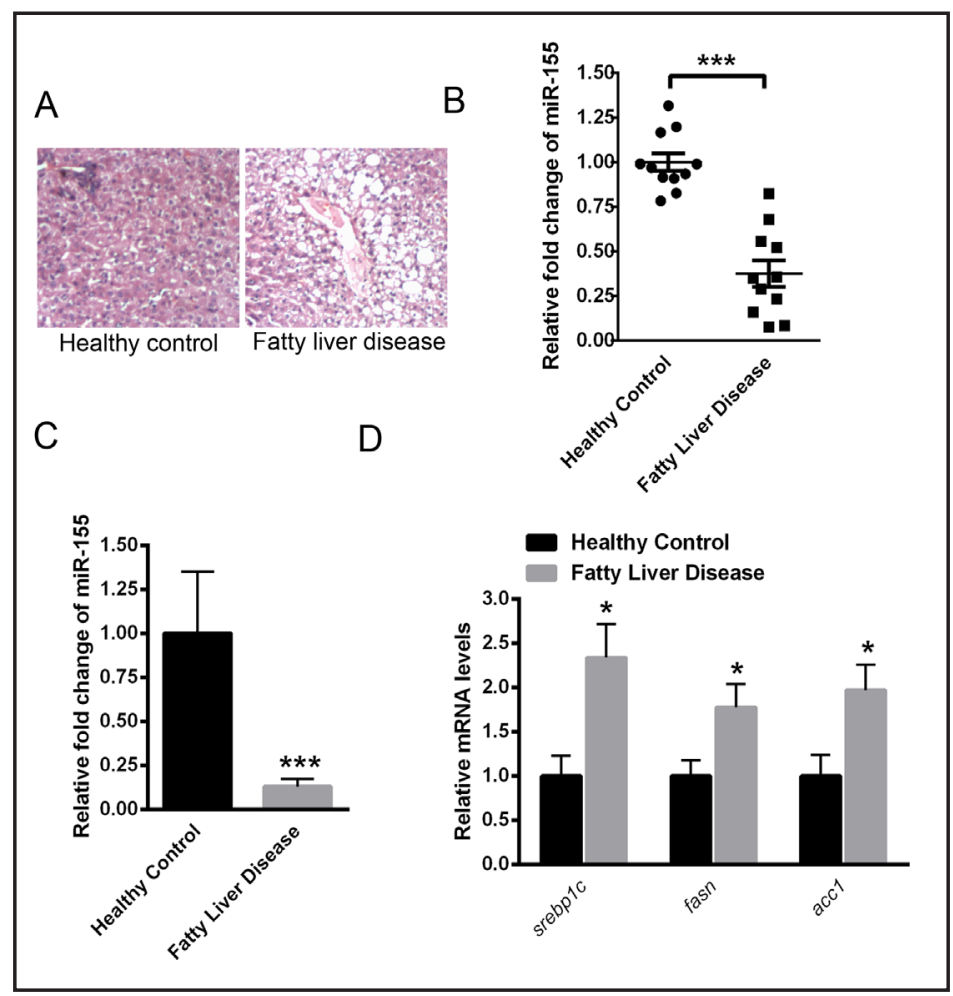

\section{KARGER}




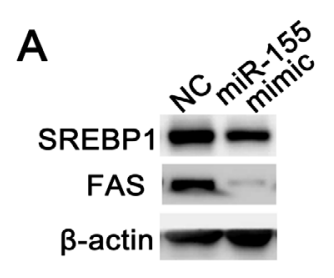

C
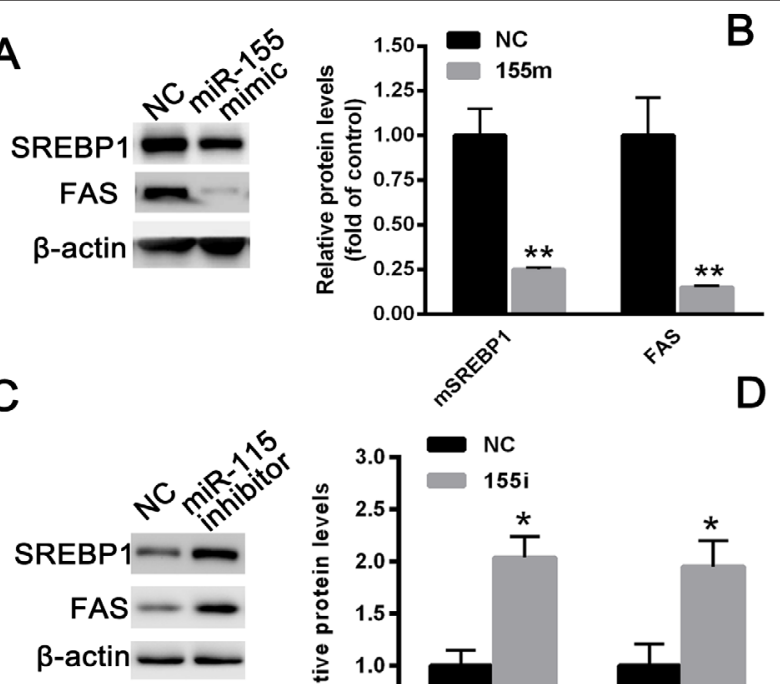
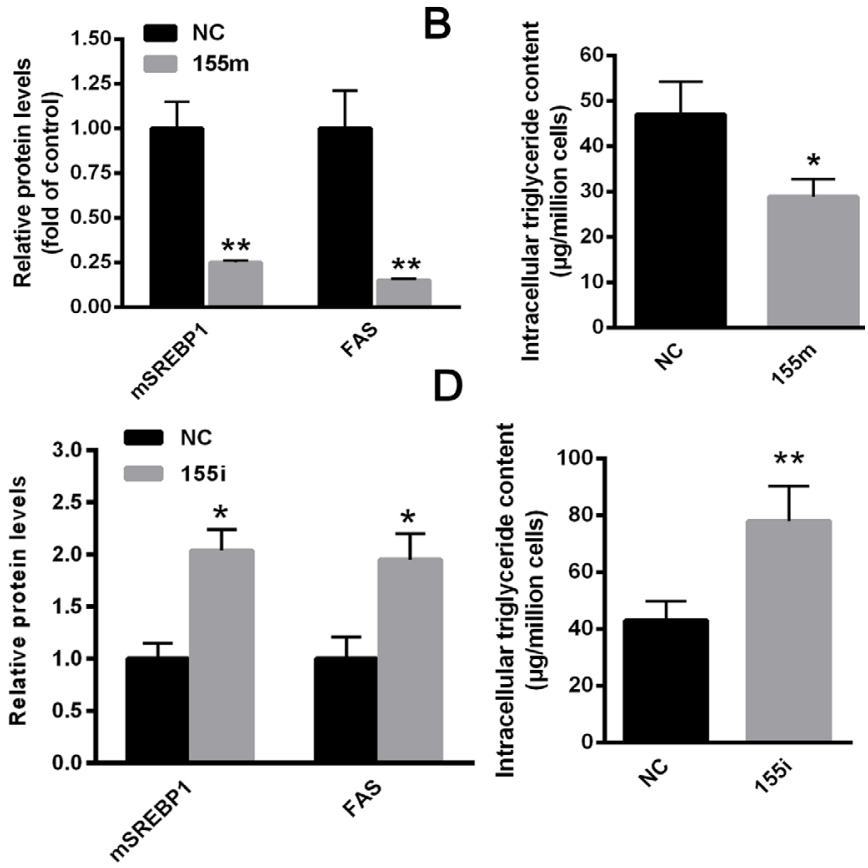

Fig. 2. Reduced miR-155 expression prompted lipogenesis in Hep1-6 cells. (A) Overexpression of miR-155 markedly suppressed SREBP1 and FAS protein levels. (B) Upregulation of miR-155 decreased the intracellular contents of lipid in Hep1-6 cells. (C) Inhibition of miR-155 significantly enhanced the expression of SREBP1 and FAS in Hep1-6 cells. (D) The intracellular contents of triglyceride were significantly increased when the level of miR-155 was suppressed. Data represent the mean \pm S.D., $n=3$ independent experiments, ${ }^{*} \mathrm{p}<0.05,{ }^{* *} \mathrm{p}<0.01$ vs. controls.

A

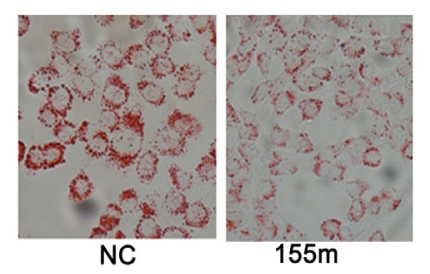

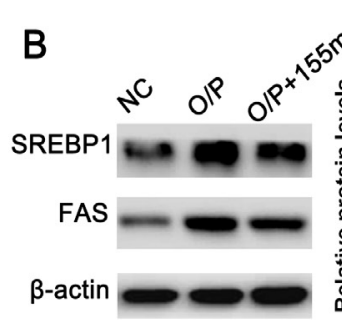

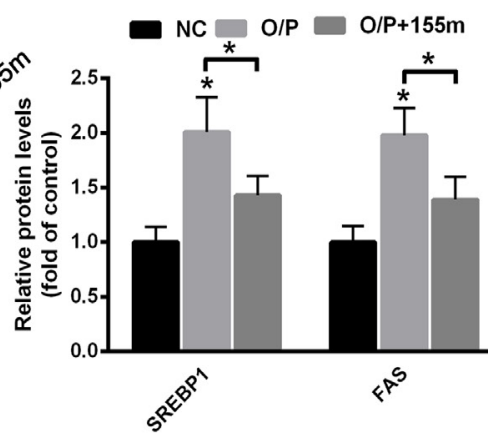

Fig. 3. Overexpression of miR-155 could abolish oleic acid/palmitic acid (O/P)-induced lipid accumulation. (A) Oil Red staining. (B) Western blot analysis of cells treated with miR-155 mimic in the presence of 0/P. Data represent the mean \pm S.D., $n=3$ independent experiments, ${ }^{*} \mathrm{p}<0.05$ vs. controls.

genes related to lipogenesis, including srebp1c, fasn, and acc1, were significantly elevated in NAFLD patients (Fig. 1D). These data suggested that reduced miR-155 and elevated LXR $\alpha$ activity may promote fatty acid synthesis in the livers of NAFLD patients.

Reduction of miR-155 contributed to abnormal upregulation of lipogenic genes

Next, we explored the role of miR-155 on lipid accumulation in Hep1-6 cells. As shown in Fig. 2A, overexpression of miR-155 markedly suppressed the protein level of SREBP1 and FAS. Meanwhile, the intracellular contents of lipids were also significantly decreased (Fig. 2B). In contrast, inhibition of miR-155 significantly enhanced the expression of SREBP1 and

\section{KARGER}




\section{Cellular Physiology and Biochemistry

Fig. 4. $L X R \alpha$ was the target gene of miR-155. (A) Prediction of a possible binding site on the 3'UTR of LXR $\alpha$ by miR-155. (B) Dual luciferase reporter assay showed that miR-155 decreased the relative luciferase activity of pmirGLO-LXR $\alpha$-3'UTR. (C) The protein level of LXR $\alpha$ was significantly suppressed in Hep1-6 cells transfected with miR-155 mimic. (D) The level of $\operatorname{LXR} \alpha$ was markedly increased in Hep1-6 cells transfected with miR-155 inhibitor. (E) Immunofluorescence analysis confirmed that upregulation of miR-155 decreased the expression of LXR $\alpha$. Data represent the mean \pm S.D., $\mathrm{n}=3$ independent experiments, * $\mathrm{p}<0.05,{ }^{* *} \mathrm{p}<0.01$ vs. controls.

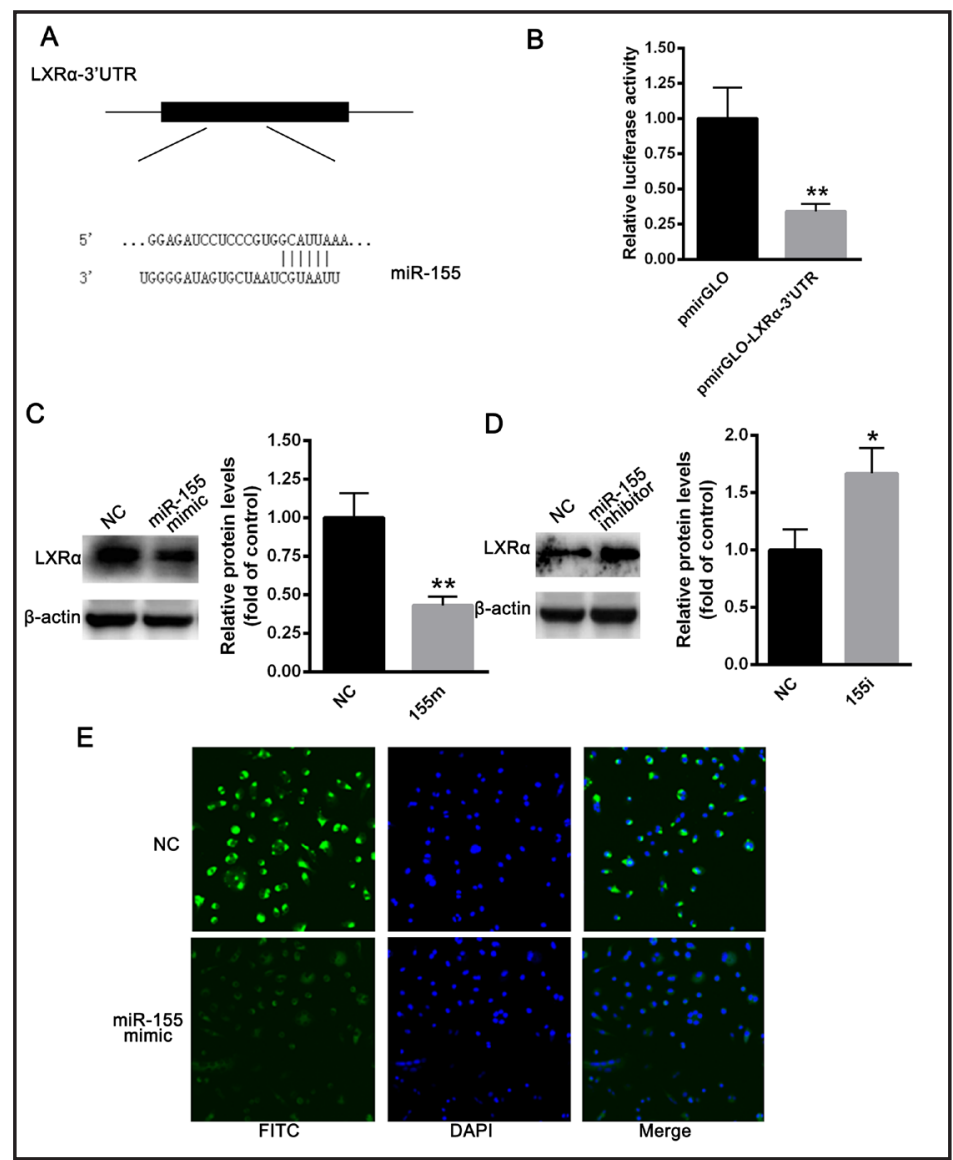

FAS in Hep1-6 cells transfected with miR-155 inhibitor (Fig. 2C). More importantly, the intracellular contents of triglyceride were significantly increased when the level of miR-155 was suppressed (Fig. 2D).

Overexpression of miR-155 could abolish oleic acid/palmitic acid (O/P)-induced lipid accumulation

To further explore whether upregulation of miR-155 ameliorates lipid accumulation, the Hep1-6 cells were pre-treated with free fatty acid, a mixture of oleic acid/ palmitic acid $(0 / P)$ with a final concentration of $300 \mu \mathrm{M}$. Large lipid droplets were shown in Hep1-6 cells pre-incubated with 0/P (Fig. 3A). In comparison, overexpression of miR-155 reduced the lipid droplets in Hep1-6 cells in the presence of O/P (Fig. 3A). Western blot analysis demonstrated that the expression of lipogenic genes, including SREPB1 and FAS, was significantly increased in Hep1-6 cells (Fig. 3B). In contrast, transfection with miR-155 mimic could partially abolish such effects (Fig. 3B).

LXR $\alpha$ was the target gene of $m i R-155$

Then, we investigated the possible target gene of miR-155 that is involved in lipogenesis. Interestingly, a conserved binding site for miR-155 on the 3'UTR of LXR $\alpha$ was identified through TargetScan (http://www.targetscan.org/vert_71/) (Fig. 4A). Then, the 3'UTR of LXR $\alpha$ was cloned into the dual luciferase reporter, pmirGLO. As shown in Figure 4B, overexpression of miR-155 significantly suppressed the relative luciferase activity of pmirGLO-LXR $\alpha-3^{\prime}$ UTR compared with the blank vector (Fig. 4B). Meanwhile, the protein level of LXR $\alpha$ was significantly suppressed in Hep1-6 cells transfected with miR-155 mimic (Fig. 4C), while the level of LXR $\alpha$ was markedly increased in Hep1-6 cells transfected with miR155 inhibitor (Fig. 4D). Immunofluorescence analysis also confirmed that upregulation of 
A
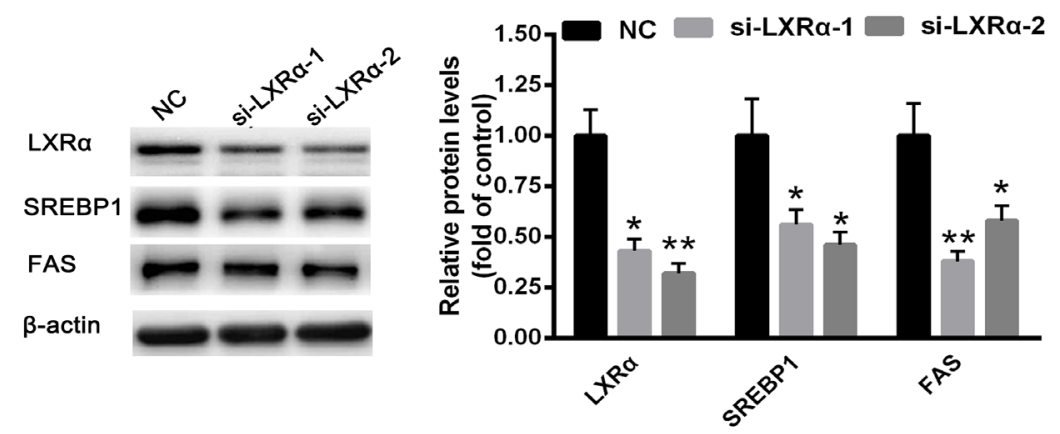

B
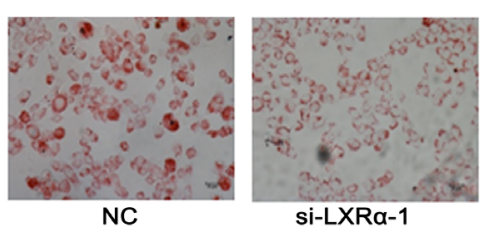

si-LXRa-1

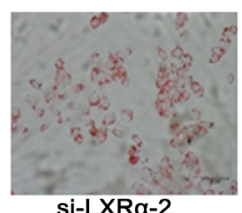

si-LXRa-2

C
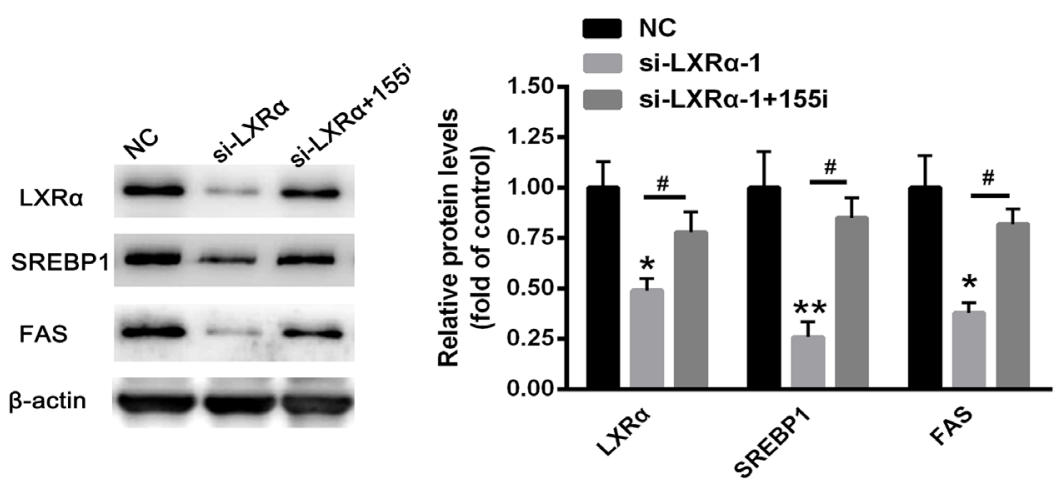

Fig. 5. Silencing of LXR $\alpha$ decreased lipid accumulation in Hep1-6 cells. (A) Knockdown of LXR $\alpha$ significantly inhibited the protein levels of SREBP1 and FAS in Hep1-6 cells. (B) Oil Red staining showed that the lipid droplets were decreased in Hep1-6 cells transfected with siRNAs targeting LXR $\alpha$. (C) The inhibition of miR155 could partially reverse LXR $\alpha$ inhibition-induced downregulation of SREBP1 and FAS in Hep1-6 cells. Data represent the mean \pm S.D., $n=3$ independent experiments, ${ }^{*} \mathrm{p}<0.05,{ }^{*} \mathrm{p}<0.01$ vs. controls; $\# \mathrm{p}<0.05$, vs. si-LXR $\alpha$.

miR-155 decreased the fluorescence of LXR $\alpha$ (Fig. 4E). These data indicated that LXR $\alpha$ was the target gene of miR-155.

\section{Silence of LXR $\alpha$ decreased lipid accumulation in Hep1-6 cells}

Furthermore, the expression of LXR $\alpha$ was silenced by two specific siRNAs. As shown in Fig. 5A, knockdown of LXR $\alpha$ significantly inhibited the protein levels of SREBP1 and FAS in Hep1-6 cells. Meanwhile, the lipid droplets were decreased in Hep1-6 cells transfected with siRNAs targeting LXR $\alpha$ (Fig. 5B). More importantly, we determined that inhibition of miR155 could partially reverse LXR $\alpha$ inhibition-induced downregulation of SREBP1 and FAS in Hep1-6 cells (Fig. 5C).

\section{Upregulation of miR-155 decreased lipid accumulation in HFD-fed mice}

Based on the in vitro study, we further extended our research into the in vivo setting. Through tail vein injection, the ad-miR-155m or ad-NC was delivered to the livers of HFD fed mice. The livers of HFD-fed mice transfected with ad-miR-155m were significantly reduced 


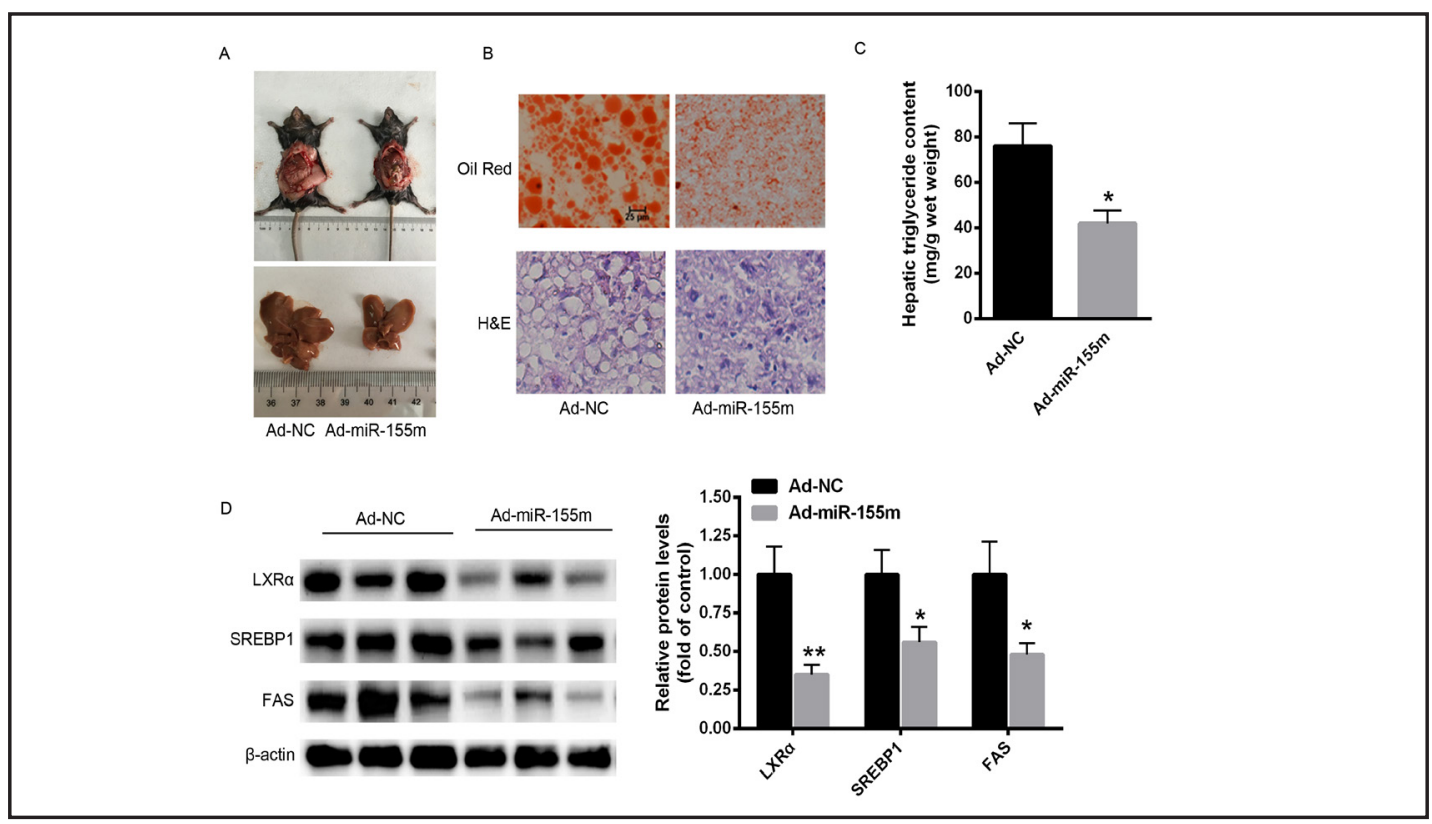

Fig. 6. Upregulation of miR-155 decreased lipid accumulation in HFD-fed mice. (A) Representative figures of the mice and their livers after injection with ad-miR-155m or ad-NC. (B) Oil Red staining and H\&E staining showed that the lipid droplets were reduced in the livers of HFD-fed mice transfected with ad-miR-155m compared with ad-NC. (C) The enhanced level of miR-155 decreased the hepatic triglyceride contents compared with ad-NC. (D) The protein levels of LXR $\alpha$, SREBP1 and FAS were significantly reduced in the livers of HFD-fed mice transfected with ad-miR-155m compared with ad-NC. Data represent the mean \pm S.D., $n=3$ independent experiments, ${ }^{*} \mathrm{p}<0.05,{ }^{* *} \mathrm{p}<0.01$ vs. controls.

compared with those transfected with ad-NC (Fig. 6A). Oil Red staining and H\&E staining showed that the lipid droplets were reduced in the livers of HFD-fed mice transfected with ad-miR-155m compared with ad-NC (Fig. 6B). Further study showed that upregulation of miR-155 decreased the hepatic triglyceride contents compared with ad-NC (Fig. 6C). Meanwhile, the protein levels of LXR $\alpha$, SREBP1 and FAS were significantly reduced in the livers of HFD-fed mice transfected with ad-miR-155m compared with ad-NC (Fig. 6D).

\section{Discussion}

At present, NAFLD is considered to be a common cause of liver damage $[19,20]$. It is reported that abnormal fat deposition in the liver may eventually develop into nonalcoholic steatohepatitis (NASH) and eventually fibrosis [21]. In the past years, researchers have been making great efforts to elucidate the pathogenesis of NAFLD. However, little is known about the underlying mechanism of NAFLD. Increasing evidence has demonstrated that miRNAs play key roles in the pathology of NAFLD $[14,22]$.

In the current study, we first demonstrated that the level of miR-155 was decreased in the livers and peripheral blood of NAFLD patients compared with those of healthy controls, suggesting that a decreased miR-155 level may be associated with the progression of NAFLD. Further study demonstrated that inhibition of miR-155 resulted in abnormal lipid accumulation in hepatocytes, whereas overexpression of miR-155 improved lipid accumulation and the expression of lipogenic genes, including SREBP1 and FAS. These data demonstrate a possible role of miR-155 as a biomarker for hepatic lipogenesis.

LXRs are nuclear receptors that widely modulate lipid metabolism, such as cholesterol and bile acids [23]. Initially, LXR was identified as a member of the nuclear receptor superfamily, and it could form an active complex with retinoid X receptor $\alpha(\mathrm{RXR} \alpha)[24,25]$. LXR $\alpha$ 


\section{Cellular Physiology Cell Physiol Biochem 2016;39:2239-2248

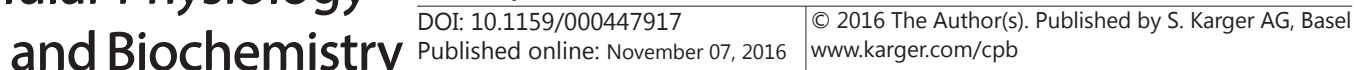 \\ Wang et al.: MiR-155 Targets LXR $\alpha$ in NAFLD Livers}

was reported to be highly expressed in liver and it is reported that certain oxysterols and intermediates in the cholesterol synthetic pathway can activate the transcription of LXR $\alpha$ [26]. SREBP1 was well known to regulate the expression of lipogenic genes, including FAS, acetyl CoA carboxylase (ACC) and stearoyl CoA desaturase-1 (SCD1) [27]. It is known that LXR $\alpha$ could bind the promoter of SREBP-1c, thereby stimulating lipid synthesis in the liver [28].

In the current study, we identified that LXR $\alpha$ was a direct target gene of miR-155. Bioinformatics prediction revealed a conserved binding site of miR-155 in the 3'UTR of LXR $\alpha$. A dual luciferase assay demonstrated that miR-155 could markedly decrease the luciferase reporter activity of pmirGLO-LXR $\alpha$-3'UTR compared with a blank vector. We showed that knockdown of LXR $\alpha$ through specific siRNAs could significantly inhibit lipid accumulation both in hepatocytes and livers. We then explored the lipid metabolism-related signaling pathway and found that SREBP1 was also suppressed when LXR was silenced. More importantly, inhibition of miR-155 could partially abolish the LXR silence-induced upregulation of lipogenic genes. These data suggested that upregulation of miR-155 improved lipid accumulation mainly by targeting LXR $\alpha$.

In summary, decreased expression of miR-155 in the peripheral blood may be utilized as a potential novel biomarker for NAFLD screening mainly by targeting LXR $\alpha$.

\section{Acknowledgement}

This study was supported by the Fundamental Research Funds for the Central Universities (021414380245), the Research Fund for the Doctoral Program of Higher Education of china (20123237120008) and the Advance Research Program of National Fund of Nanjing University of Chinese Medicine(14XXY06).

\section{Disclosure Statement}

We declare no conflicts of interest.

\section{References}

1 An BQ, Lu LL, Yuan C, Xin YN, Xuan SY: Leptin receptor gene polymorphisms and the risk of non-alcoholic fatty liver disease and coronary atherosclerosis in the chinese han population. Hepat Mon 2016;16:e35055.

2 Aguilar-Olivos NE, Carrillo-Cordova D, Oria-Hernandez J, Sanchez-Valle V, Ponciano-Rodriguez G, Ramirez-Jaramillo M, Chable-Montero F, Chavez-Tapia NC, Uribe M, Mendez-Sanchez N: The nuclear receptor fxr, but not lxr, up-regulates bile acid transporter expression in non-alcoholic fatty liver disease. Ann Hepatol 2015;14:487-493.

3 Bieghs V, Van Gorp PJ, Wouters K, Hendrikx T, Gijbels MJ, van Bilsen M, Bakker J, Binder CJ, Lutjohann D, Staels B, Hofker MH, Shiri-Sverdlov R: Ldl receptor knock-out mice are a physiological model particularly vulnerable to study the onset of inflammation in non-alcoholic fatty liver disease. PLoS One 2012; 7:e30668.

4 Ducheix S, Montagner A, Theodorou V, Ferrier L, Guillou H: The liver x receptor: A master regulator of the gut-liver axis and a target for non alcoholic fatty liver disease. Biochem Pharmacol 2013;86:96-105.

$5 \quad$ Fuchs M: Non-alcoholic fatty liver disease: The bile acid-activated farnesoid x receptor as an emerging treatment target. J Lipids 2012;2012:934396.

6 Ikegami T, Hyogo H, Honda A, Miyazaki T, Tokushige K, Hashimoto E, Inui K, Matsuzaki Y, Tazuma S: Increased serum liver $\mathrm{x}$ receptor ligand oxysterols in patients with non-alcoholic fatty liver disease. J Gastroenterol 2012;47:1257-1266. 


\section{Cellular Physiology Cell Physiol Biochem 2016;39:2239-2248 and Biochemistry Published online: November 07, 2016 \begin{tabular}{l|l} 
DOI: 10.1159/000447917 & $\begin{array}{l}\text { (c) } 2016 \text { The Author(s). Published by S. Karger AG, Basel } \\
\text { www.karger.com/cpb }\end{array}$
\end{tabular} \\ Wang et al.: MiR-155 Targets LXR $\alpha$ in NAFLD Livers}

7 Lee J, Hong SW, Rhee EJ, Lee WY: Glp-1 receptor agonist and non-alcoholic fatty liver disease. Diabetes Metab J 2012;36:262-267.

8 Shi L, Shi L, Zhang H, Hu Z, Wang C, Zhang D, Song G: Oxymatrine ameliorates non-alcoholic fatty liver disease in rats through peroxisome proliferator-activated receptor-alpha activation. Mol Med Rep 2013;8:439-445.

9 Fan A, Wang Q, Yuan Y, Cheng J, Chen L, Guo X, Li Q, Chen B, Huang X, Huang Q: Liver x receptor-alpha and mir-130a-3p regulate expression of sphingosine 1-phosphate receptor 2 in human umbilical vein endothelial cells. Am J Physiol Cell Physiol 2016;310:C216-226.

10 Qadir XV, Chen W, Han C, Song K, Zhang J, Wu T: Mir-223 deficiency protects against fas-induced hepatocyte apoptosis and liver injury through targeting insulin-like growth factor 1 receptor. Am J Pathol 2015;185:3141-3151.

11 Yang F, Hu Y, Liu HX, Wan YJ: Mir-22-silenced cyclin a expression in colon and liver cancer cells is regulated by bile acid receptor. J Biol Chem 2015;290:6507-6515.

12 Cheng X, Xi QY, Wei S, Wu D, Ye RS, Chen T, Qi QE, Jiang QY, Wang SB, Wang LN, Zhu XT, Zhang YL: Critical role of mir-125b in lipogenesis by targeting stearoyl-coa desaturase-1 (scd-1). J Anim Sci 2016;94:65-76.

13 Meng X, Guo J, Fang W, Dou L, Li M, Huang X, Zhou S, Man Y, Tang W, Yu L, Li J: Liver microrna-291b-3p promotes hepatic lipogenesis through negative regulation of adenosine 5 '-monophosphate (amp)-activated protein kinase alpha1. J Biol Chem 2016;291:10625-10634.

14 Feng YY, Xu XQ, Ji CB, Shi CM, Guo XR, Fu JF: Aberrant hepatic microrna expression in nonalcoholic fatty liver disease. Cell Physiol Biochem 2014;34:1983-1997.

15 Tang B, Lei B, Qi G, Liang X, Tang F, Yuan S, Wang Z, Yu S, He S: Microrna-155-3p promotes hepatocellular carcinoma formation by suppressing fbxw7 expression. J Exp Clin Cancer Res 2016;35:93.

16 Feng JA: Target research on tumor biology characteristics of mir-155-5p regulation on gastric cancer cell. Pak J Pharm Sci 2016;29:711-718.

17 Yang S, Li F, Jia S, Zhang K, Jiang W, Shang Y, Chang K, Deng S, Chen M: Early secreted antigen esat-6 of mycobacterium tuberculosis promotes apoptosis of macrophages via targeting the microrna155-socs1 interaction. Cell Physiol Biochem 2015;35:1276-1288.

$18 \mathrm{Ng} \mathrm{R}$, Wu H, Xiao H, Chen X, Willenbring H, Steer CJ, Song G: Inhibition of microrna-24 expression in liver prevents hepatic lipid accumulation and hyperlipidemia. Hepatology 2014;60:554-564.

19 Yang ZX, Shen W, Sun H: Effects of nuclear receptor fxr on the regulation of liver lipid metabolism in patients with non-alcoholic fatty liver disease. Hepatol Int 2010;4:741-748.

20 Yavas 0, Eren 00: Peroxisome proliferator-activated receptor gamma: A common link for non-alcoholic fatty liver disease and colorectal cancer? Med Hypotheses 2007;69:947.

21 Brunt EM: Pathology of nonalcoholic fatty liver disease. Nat Rev Gastroenterol Hepatol 2010;7:195-203.

22 Hu X, Zhang F, Liu XR, Wu YT, Ni YM: Efficacy and potential microrna mechanism for computed tomography-guided percutaneous radiofrequency ablation of primary lung cancer and lung metastasis from liver cancer. Cell Physiol Biochem 2014;33:1261-1271.

$23 \mathrm{Xu}$ JY, Li ZP, Zhang L, Ji G: Recent insights into farnesoid x receptor in non-alcoholic fatty liver disease. World J Gastroenterol 2014;20:13493-13500.

24 Willy PJ, Umesono K, Ong ES, Evans RM, Heyman RA, Mangelsdorf DJ: Lxr, a nuclear receptor that defines a distinct retinoid response pathway. Genes Dev 1995;9:1033-1045.

25 Harasiuk D, Baranowski M, Zabielski P, Chabowski A, Gorski J: Lxr agonist t0901317-induced hyperlipidemia does not lead to lipid accumulation in the rat heart. Cell Physiol Biochem 2015;35:1095-1106.

26 Janowski BA, Grogan MJ, Jones SA, Wisely GB, Kliewer SA, Corey EJ, Mangelsdorf DJ: Structural requirements of ligands for the oxysterol liver x receptors lxralpha and lxrbeta. Proc Natl Acad Sci U S A 1999;96:266-271.

27 Hong C, Tontonoz P: Liver x receptors in lipid metabolism: Opportunities for drug discovery. Nat Rev Drug Discov 2014;13:433-444.

28 Korach-Andre M, Gustafsson JA: Liver x receptors as regulators of metabolism. Biomol Concepts 2015;6:177-190. 